Supporting Information for:

\title{
Structural Analysis of Bottlebrush Block Copolymer Micelles Using Small-Angle X-ray Scattering
}

Seyoung Kim ${ }^{1}$, Yunshik Cho ${ }^{1}$, Jee Hyun Kim ${ }^{1}$, Sanghoon Song ${ }^{3}$, Jeewoo Lim ${ }^{3}$, Soo-Hyung Choi $^{*}, 2$, and Kookheon Char ${ }^{*}, 1$

${ }^{1}$ Department of Chemical and Biological Engineering, Seoul National University, Gwanak-gu, Seoul 08826, Republic of Korea

${ }^{2}$ Department of Chemical Engineering, Hongik University, Mapo-gu, Seoul 04066, Republic of Korea

${ }^{3}$ Department of Chemistry, Kyung Hee University, Dongdaemun-gu, Seoul 02447, Republic of Korea

*Corresponding authors: S.C. (shchoi@hongik.ac.kr) and K.C. (khchar@snu.ac.kr) 


\section{Experimental Section}

\section{A. Methods}

Materials. Grubbs 3rd generation catalyst, N-Cyclohexyl-exo-norbornene-5,6-dicarboxyimide (ChNDI), and 5-(perfluorooctyl)norbornene (NB8F) were synthesized using previously established procedures. ${ }^{1}$ Anhydrous dichloromethane (DCM) and $\alpha, \alpha, \alpha$-trifluorotoluene (TFT) were purchased from Sigma-Aldrich and degassed through three freeze-pump-thaw cycles directly before use. All homopolymers and diblock copolymers pNB8Fblock-pChNDI (hereafter noted as PF- $b$-PC) were prepared using previously reported ring-opening metathesis polymerization (ROMP) technique, except for PF homopolymers of which polymerization reaction was carried out in TFT. Tetrahydrofuran (THF) was purchased from Sigma-Aldrich and used without further purification.

Characterization. The polymers were characterized by ${ }^{1} \mathrm{H}$ nuclear magnetic resonance spectroscopy (NMR), size exclusion chromatography (SEC), matrix-assisted laser desorption/ionization time-of-flight mass spectroscopy (MALDI), and elemental analysis (EA). The molecular weight and dispersity of PF homopolymer $(N=25)$ and PC homopolymer $(N=50)$ were estimated from MALDI (Voyager DE-STR mass spectrometer, Applied Biosystems) using 2,5-dihydroxybenzoic acid (DHB) as a matrix substance. The molecular weight and dispersity $\left(M_{w} / M_{n}\right)$ of PC homopolymers with different molecular weights were also estimated from SEC (YL9100 HPLC, YL Instrument) using THF as an eluent. The complete conversion of ROMP for both kinds of homopolymers, as well as for the block copolymers, was confirmed by the absence of monomer residues from NMR spectra (Ascend 400, Bruker). All PF- $b$-PC block copolymers were repeatedly precipitated in a mixture of toluene and petroleum ether (3:1 by volume) and re-dissolved several times before characterization and sample preparation in order to eliminate small portions of "dead" PC homopolymers (at which the propagation of the PF block was absent) from the copolymers. After purification, the elemental weight fractions of carbon and hydrogen was measured for PF- $b$-PC from EA (TruSpec Micro elemental analyzer, LECO), which were used for estimation of the molecular composition. Since there is no cosolvent for PF- $b$-PC block copolymers and most of the polymers have quite large $M_{\mathrm{n}}(>50 \mathrm{kDa})$, the molecular weight distribution of PF- $b$-PC could not be directly measured from either SEC or MALDI; therefore we assumed that each PC and PF block in PF- $b$-PC has similar dispersity $\left(M_{\mathrm{w}} / M_{\mathrm{n}}\right)$ with those of PC and PF $\left(M_{\mathrm{w}} / M_{\mathrm{n}}=1.02\right.$ and 1.05 , respectively, from MALDI measurement $)$ of which molecular weight distributions were very narrow. The results are summarized in Table 1 (in the manuscript), Table S2, and Table S3. 
Surface energy of PF was characterized by contact angle analysis. Silicon wafer substrates were sonicated in ethanol for 10 minutes, and glass substrates were cleaned by submerging in Piranha solution for 20 minutes prior to use. $5 \mathrm{w} / \mathrm{v} \%$ solution of PF $(N=500)$ in TFT was spin coated at $3000 \mathrm{rpm}$ for 60 seconds to render a PF film with flat surface on substrate and annealed overnight in vacuum oven. Sessile droplets of deionized water and several non-polar test liquids (of which volumes were 5 and $2 \mu \mathrm{L}$, respectively) were carefully loaded on the annealed PF films, and the contact angles were measured using drop shape analyzer (DSA 100, Kruss). The polar and dispersive surface energies of PF was calculated from measured contact angles using the method according to $\mathrm{Wu}$ et al. ${ }^{2}$ Also, the critical surface energy of PF was measured from contact angles of test liquids with varying dispersive surface energies. Then, Hildebrand solubility parameter $(\delta)$ for PF was estimated using the surface energy values. Finally, Flory-Huggins interaction parameter between PF and THF was calculated using $\chi=$ $0.34+\frac{v_{0}}{R T}\left(\delta_{\text {polymer }}-\delta_{\text {solvent }}\right)^{2}$, where $v_{\mathrm{o}}$ is the reference volume (which is set equal to the molar volume of THF) and $\mathrm{R}$ is the ideal gas constant. The results are summarized in Table S5.

Small-Angle X-ray Scattering (SAXS). $1 \mathrm{wt} \%$ polymer solutions of PF- $b$-PC were prepared by dissolving the polymers in THF, which is a selective solvent for PC block. The polymer solutions were equilibrated for $24 \mathrm{hr}$ at $60{ }^{\circ} \mathrm{C}$ in tightly sealed vials to bring the block copolymer micelles into the equilibrium state. The solvent evaporation was minimal $(<1 \%)$ during the equilibration. The polymer solutions were then cooled down and syringe filtered before use. SAXS experiments were conducted using 9A beamline at Pohang Accelerator Laboratory (S. Korea). Radiation of $20 \mathrm{keV}$, corresponding to a wavelength $\lambda=0.620 \AA$, was selected from an undulator beam using a double-crystal monochromator, and the sample-to-detector distance (SDD) was $6.5 \mathrm{~m}$. Samples were loaded in quartz capillaries and mounted in a thermostat stage at $25^{\circ} \mathrm{C}$, followed by X-ray exposure for 30 s. 2-D SAXS images were collected and azimuthally averaged using the data reduction software SAXSLee. The solvent background was also collected and subtracted from the solution data. The resulting data were calibrated using a glassy carbon reference sample. The scaled data were analyzed with a fitting model described elsewhere,$^{2}$ using IGOR Pro. X-ray scattering length density of each component required for SAXS analysis was estimated by the method which is described in the following section.

Transmission Electron Microscopy (TEM). Polymer solutions were prepared with the identical procedure with those for SAXS experiments. After cooling to room temperature, these were further diluted with THF to make $0.5 \mathrm{mg} / \mathrm{mL}$. Each $2 \mu \mathrm{L}$ of diluted solution was deposited on the grids with carbon support films (Electron 
Microscopy Sciences), blotted with filter paper, and finally let dried in desiccator. This procedure ensured fast evaporation and protected samples from being disrupted by moisture condensation during the evaporation. TEM images of fully dried samples were collected using LIBRA 120 (Carl Zeiss).

Static Light Scattering (SLS). Polymer solutions of different concentrations $(0.4-2.0 \mathrm{mg} / \mathrm{mL})$ were prepared with the identical procedure with those for SAXS experiments. SLS experiments were conducted using BI-200SM research goniometer (Brookhaven). Scattered light of a wavelength $\lambda=639 \mathrm{~nm}$ from the samples at $25{ }^{\circ} \mathrm{C}$ with scattering angles from $30^{\circ}$ to $110^{\circ}$ were collected. Incremental refractive indices $(\mathrm{d} n / \mathrm{d} c)$ from a series of PF- $b$-PC in THF were separately measured from differential refractometer (YL9170, YL Instrument) and employed for the evaluation of the SLS data.

Dynamic Light Scattering (DLS). Samples with different concentrations $(0.67-2.0 \mathrm{mg} / \mathrm{mL})$ were prepared with the identical composition and procedure with those for SAXS experiments. DLS experiments were conducted using Zetasizer Nano ZS90 (Malvern Instruments). Scattering data with a wavelength $\lambda=532 \mathrm{~nm}$ and a fixed scattering angle of $90^{\circ}$ was collected and the resulting time correlation functions were analyzed by cumulant method. In a separate multi-angle experiment, DLS from the PF- $b$-PC sample solutions of $1.0 \mathrm{mg} / \mathrm{mL}$ was conducted using BI-200SM research goniometer (Brookhaven) with a wavelength $\lambda=639 \mathrm{~nm}$ and scattering angles of from $30^{\circ}$ to $120^{\circ}$.

Small-Angle Neutron Scattering (SANS). SANS experiments were performed at 40mSANS beamline at HANARO Facility, Korea Atomic Energy Research Institute (S. Korea) to estimate neutron and X-ray scattering length density (SLD) for individual PF and PN blocks. Samples of $\mathrm{PC}_{500}$ and $\mathrm{PF}_{180}-b-\mathrm{PC}_{300}$ were examined at HANARO, where an instrument configuration with a wavelength $\lambda=6 \AA$, a wavelength spread $(\Delta \lambda / \lambda)$ of 0.12 , a SDD of $11.5 \mathrm{~m}$ was chosen. Samples were loaded in the $2 \mathrm{~mm}$ demountable cells and placed in a temperaturecontrolled heating block at $25^{\circ} \mathrm{C}$. Subsequently, scattering intensities were collected by a 2-D detector for $30 \mathrm{~min}$. The 2-D scattering images were azimuthally averaged, reduced to an absolute scale, and background subtracted using the package provided by HANARO based on IGOR Pro. 


\section{B. Scattering Length Densities (SLDs) and Scattering Model}

Measurements of X-ray SLDs by solvent contrast variation. Knowing neutron or X-ray scattering length densities (SLDs, $\left.\rho_{\mathrm{n}}\right)$ is necessary in any analysis for bi-component structures dispersed in the media. It is possible to calculate $\rho_{\mathrm{n}}$ for any materials when the gravimetric density $\left(\rho_{\mathrm{g}}\right)$ is known. Still, accurate determination of $\rho_{\mathrm{g}}$ for powdery or highly viscous polymer is not a trivial task, which is the case for our newly synthesized PN and PC polymers. Thus, we directly measured $\rho_{\mathrm{n}}$ of the materials with the solvent contrast variation.

First, PC homopolymer $(N=500)$ was synthesized and diluted in mixture of (hydrogenated) h-toluene and (deuterated) d-toluene to yield $5 \mathrm{wt} \%$ solutions with 4 different solvent $\operatorname{SLDs}\left(\rho_{\mathrm{n}, \mathrm{s}}\right)$. The $\operatorname{SLD}$ of PC $\left(\rho_{\mathrm{n}, \mathrm{PC}}\right)$ could be determined from the fact that the scattering intensity, $I(q)$, is proportional to $\left(\Delta \rho_{\mathrm{hPC}}\right)^{2}$ where $q$ is the scattering vector and $\Delta \rho_{i}$ is the residual SLD for $i$-th phase $\left(\Delta \rho_{i}=\rho_{\mathrm{n}, i}-\rho_{\mathrm{n}, \mathrm{s}}\right)$. A linear plot of $\left[I_{q \rightarrow 0}\right]^{1 / 2}$ vs $\rho_{\mathrm{n}, \mathrm{s}}$ was constructed to visualize the relationship where $I_{q \rightarrow 0}$ is the scattering intensity at the limit of zero angle $(q \rightarrow 0)$ (Figure S1a). It is easily noticed that $\rho_{\mathrm{n}, \mathrm{PC}}$ should be same with $\rho_{\mathrm{n}, \mathrm{s}}$ at the x-intercept (i.e., $\Delta \rho_{i}=0$ ).

Knowing $\rho_{\mathrm{n}, \mathrm{PF}}$ is trickier because PF homopolymer is not soluble in organic solvents with high hydrogen content (e.g., hexane, toluene), which does not allow simple solvent contrast variation experiment. Therefore, we instead used $\mathrm{PF}_{180}-b-\mathrm{PC}_{300}$ block copolymers diluted in mixture of h-THF and d-THF to yield $1 \mathrm{wt} \%$ solutions with 5 different $\rho_{\mathrm{n}, \mathrm{s}}$. The scattering models for such micelles are discussed in detail in the following section. For the simplicity, only $I_{0}$ for different $\rho_{\mathrm{n}, \mathrm{s}}$ was evaluated and analyzed by the fact that

$$
I_{q \rightarrow 0} \sim\left(f_{\mathrm{PF}} \Delta \rho_{\mathrm{PF}}+f_{\mathrm{PC}} \Delta \rho_{\mathrm{PC}}\right)^{2}
$$

where $f_{i}$ is the volume composition of $i$-th block in whole block copolymer Again, a linear plot of $\left[I_{q \rightarrow 0}\right]^{1 / 2}$ vs $\rho_{\mathrm{n}, \mathrm{s}}$ was constructed (Figure S1b). From the $\rho_{\mathrm{n}, \mathrm{s}}$ value at the x-intercept, $\rho_{\mathrm{n}, \mathrm{PF}}$ was determined by the following equation:

$$
\rho_{\mathrm{n}, \mathrm{PF}}=f_{\mathrm{PF}}^{-1}\left(\rho_{\mathrm{n}, \mathrm{s}}-f_{\mathrm{PC}} \rho_{\mathrm{PC}}\right)
$$

$f_{i}$ was calculated from the knowledge of the number of repeat units and gravimetric density of each block. In fact, the analysis using eq. 2 retains some self-consistent fashion because only presumed $\rho_{\mathrm{n}, \mathrm{PF}}$ enables the calculation of $\rho_{\mathrm{g}, \mathrm{PF}}$ and $f_{\mathrm{PF}}$ further. Through such analysis, every information was obtained from the solvent contrast variation studies and listed on Table S1. It is noted that separate density measurement using mercury intrusion porosimetry gives $\rho_{\mathrm{g}, \mathrm{PF}} \simeq 1.7$, which agrees well with the results from SLD measurement. 

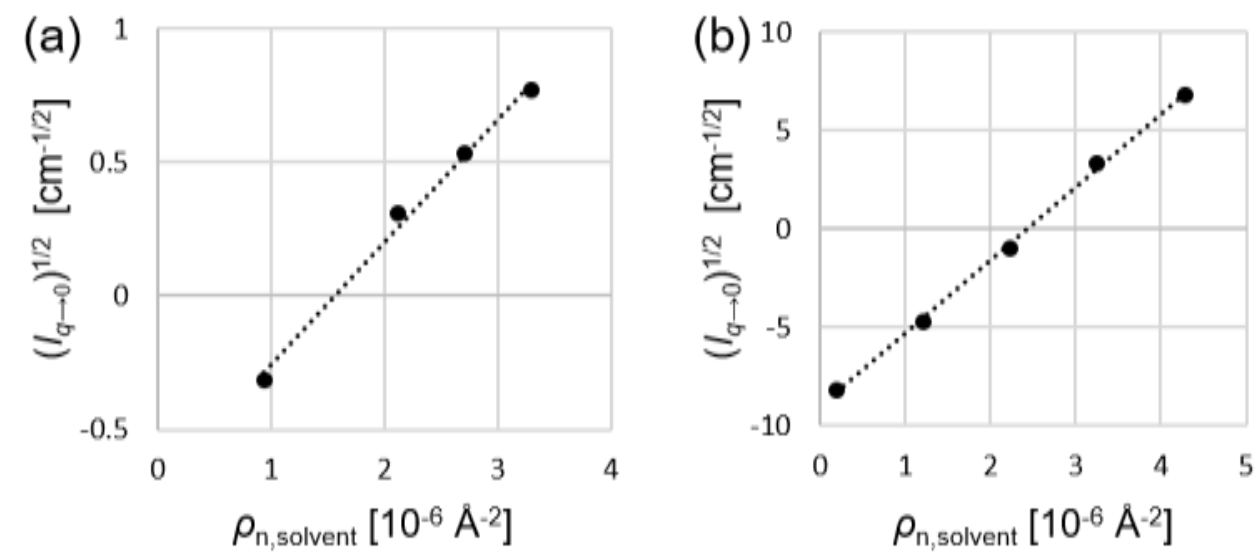

Figure S1. SANS intensities extrapolated to the zero angle $(q \rightarrow 0)$ plotted as functions of solvent SLDs. The dotted lines are linear fits. (a) $5 \mathrm{wt} \%$ solutions of $\mathrm{PC}_{500}$ homopolymer and (b) $1 \mathrm{wt} \%$ solutions of $\mathrm{PF}_{180}-b-\mathrm{PC}_{300}$ block copolymer were used, respectively.

Table S1. Neutron SLDs, X-ray SLDs, and gravimetric densities.

\begin{tabular}{cccc}
\hline Component & $\rho_{\mathrm{n}}\left[10^{-6} \AA^{-2}\right]$ & $\rho_{\mathrm{x}}\left[10^{-6} \AA^{-2}\right]$ & $\rho_{\mathrm{g}}[\mathrm{g} / \mathrm{ml}]$ \\
\hline $\mathrm{PF}$ & 3.43 & 15.0 & 1.80 \\
$\mathrm{PC}$ & 1.56 & 11.7 & 1.28 \\
\hline
\end{tabular}


Small-Angle Scattering Model for Block Copolymer Micelles. The SAXS profiles of PF- $b$-PC micelles were adjusted by the detailed fitting model given by Pedersen et al., ${ }^{2}$ where the micelles are modelled as spherical cores and polymer brushes attached to the core surface. All contributions from the core and the corona chains which give rise to the scattering from a single micelle are considered to model the scattering form factor for a micelle, $P_{\text {mic }}(q)$, which gives

$$
\begin{aligned}
P_{\text {mic }}(q)= & {\left[N_{\text {agg }} \beta_{\text {corona }} A_{\text {core }}(q)\right]^{2}+2 N_{\text {agg }}^{2} \beta_{\text {core }} \beta_{\text {corona }} A_{\text {core }}(q) A_{\text {corona }}(q)+N_{\text {agg }} \beta_{\text {corona }}{ }^{2} P_{\text {corona }}(q)+} \\
& N_{\text {agg }}\left(N_{\text {agg }}-1\right)\left[\beta_{\text {corona }} A_{\text {corona }}(q)\right]^{2}
\end{aligned}
$$

where $q$ is the scattering vector, $N_{\text {agg }}$ the aggregation number, and $\beta_{i}$ total excess scattering length of species $i$ ( $i$ denotes either the core or the corona block) defined as $\beta_{i}=v_{i}\left(\rho_{i}-\rho_{\text {solvent }}\right)$. Here, $v_{i}$ is the molecular volume of the chain $i$ and $\rho_{i}$ is the scattering length densities (SLDs) of species $i$.

The first term in eq 3 is the self-correlation of the spherical core with radius $R_{\text {core }}$ and width of the corecorona interface $\sigma_{\text {int }}$ at which SLD smoothly decays. The normalized form factor of the core, $A_{\text {core }}(q)^{2}$, is given as

$$
A_{\text {core }}(q)^{2}=\Phi\left(q R_{\text {core }}\right)^{2} \exp \left(-q^{2} \sigma_{\text {int }}^{2}\right)
$$

where $\Phi(x)=3 x^{-3}(\sin x-x \cos x)$, the Fourier transformed density function. The second term in eq 3 is the crosscorrelation between the core and the corona chains, where the normalized Fourier transform of the radial density distribution function of the corona $\left(\phi_{\text {corona }}(r)\right)$ is given as

$$
A_{\text {corona }}(q)=\frac{4 \pi \int \phi_{\text {corona }}(r) \frac{\sin (q r)}{q r} r^{2} d r}{4 \pi \int \phi_{\text {corona }}(r) r^{2} d r} \exp \left(-q^{2} \sigma_{\text {int }}^{2} / 2\right)
$$

assuming radial symmetry of the corona density profile. A number of models for $\phi_{\text {corona }}(r)$ have been proposed to describe the real behavior of micelles under various conditions. Here, a linear combination of two cubic $b$ spline functions with two fitting parameters was chosen, following the works by Pedersen et al. ${ }^{2}$ The last two terms in eq 3 are the self- and cross-correlations within the corona domain, where the chain form factor, $P_{\text {corona }}(q)$, is approximated by the Debye function for a Gaussian chain with radius of gyration $R_{\mathrm{g}}$,

$$
P_{\text {corona }}(q)=2 x^{-2}(\exp (-x)-1+x)
$$

with $x=q^{2} R_{\mathrm{g}}{ }^{2}$.

The total scattering intensity is obtained using the form factor of micelle and the hard-sphere structure factor $(S(q))$,

$$
I(q)=P_{\text {mic }}(q)+A_{\operatorname{mic}}(q)^{2}[S(q)-1]
$$

where $A_{\text {mic }}(q)$ is the form factor amplitude of the radial scattering length distribution given by

$$
A_{\text {mic }}(q)=N_{\text {agg }}\left[\beta_{\text {core }} A_{\text {core }}(q)+\beta_{\text {corona }} A_{\text {corona }}(q)\right]
$$


It is noted that the structure factor brings two additional fitting parameters, namely, the hard sphere radius $\left(R_{\mathrm{hs}}\right)$ and volume fraction $\left(\eta_{\mathrm{hs}}\right)$. Furthermore, a Gaussian distribution for the core radius, $D\left(R_{\text {core }}\right)$, is employed to account for polydispersity in micelle size. The total scattering intensity for the polydisperse model is given as

$$
I(q)=\int D\left(R_{\text {core }}\right)\left\{P_{\text {mic }}(q)+A_{\text {mic }}(q)^{2}[S(q)-1]\right\} \mathrm{d} R_{\text {core }}
$$

where the Gaussian distribution $D\left(R_{\text {core }}\right)$ is parameterized with the average radius $\left\langle R_{\text {core }}\right\rangle$ and the standard deviation $\sigma_{\mathrm{R}}$ and truncated at $R_{\text {core }}=0$ for the calculation. Eq 9 was used to fit the absolute-scaled SAXS data. 


\section{Molecular Characterizations}

A. NMR

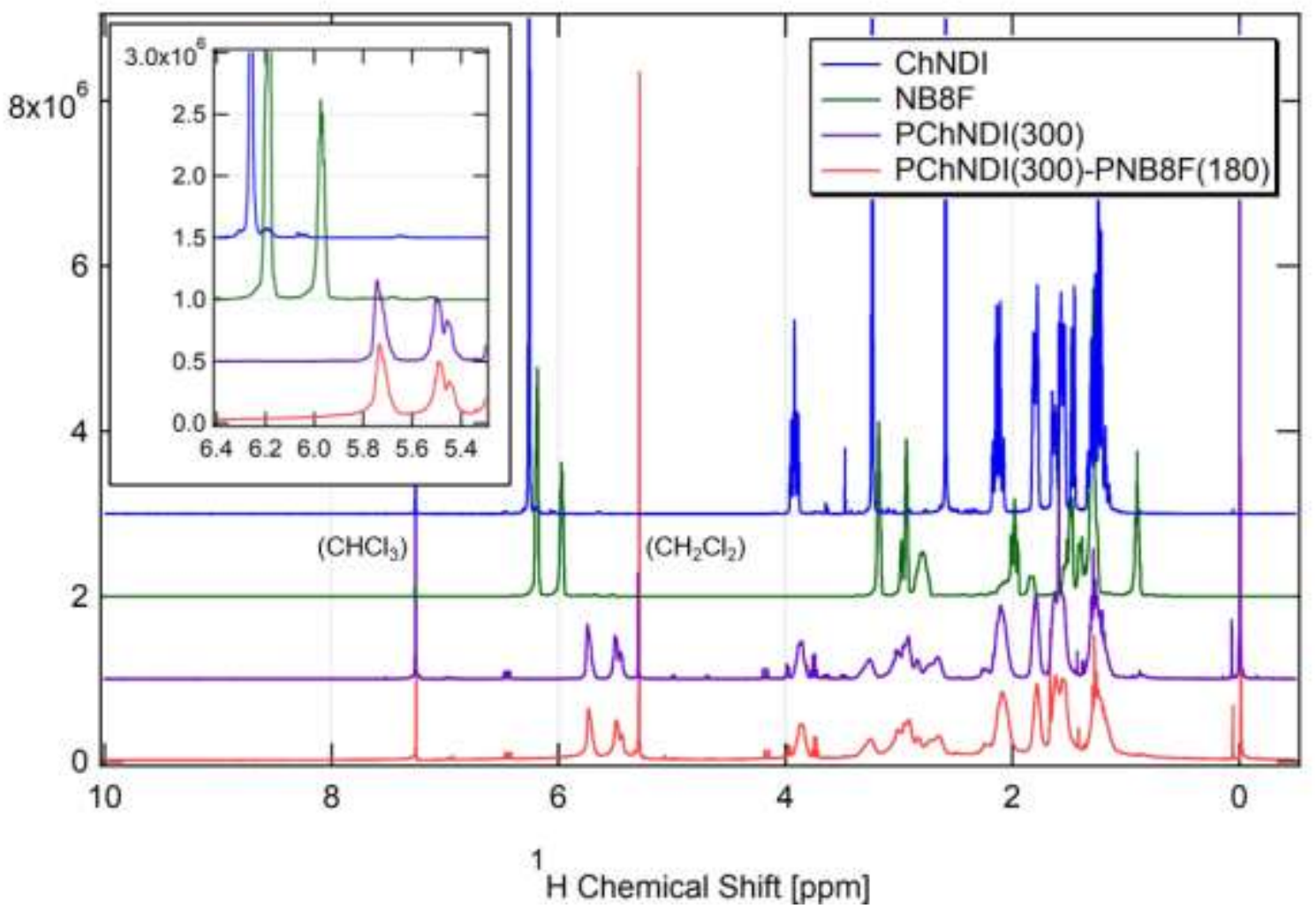

Figure S2. The ${ }^{1} \mathrm{H}$ NMR spectra of aliquots from the reaction mixtures after polymerization of PC300 and PF180$b$ - $\mathrm{PC} 300 \mathrm{H}$ in $\mathrm{CDCl}_{3}$ at $25^{\circ} \mathrm{C}$. The spectra from their monomers ChNDI and NB8F, obtained at the same condition, were also displayed for comparison. The denoted peaks indicate solvent residues. (Inset) Magnified display over the observed region of double bonds $(-\mathrm{HC}=\mathrm{CH}-)$ shows that the monomers $(6.3-5.9 \mathrm{ppm})$ in all reaction mixtures were completely consumed (conversion $>99.5 \%$ ) during the ring-opening metathesis polymerization (ROMP) employed for the synthesis of block copolymers. 
B. SEC

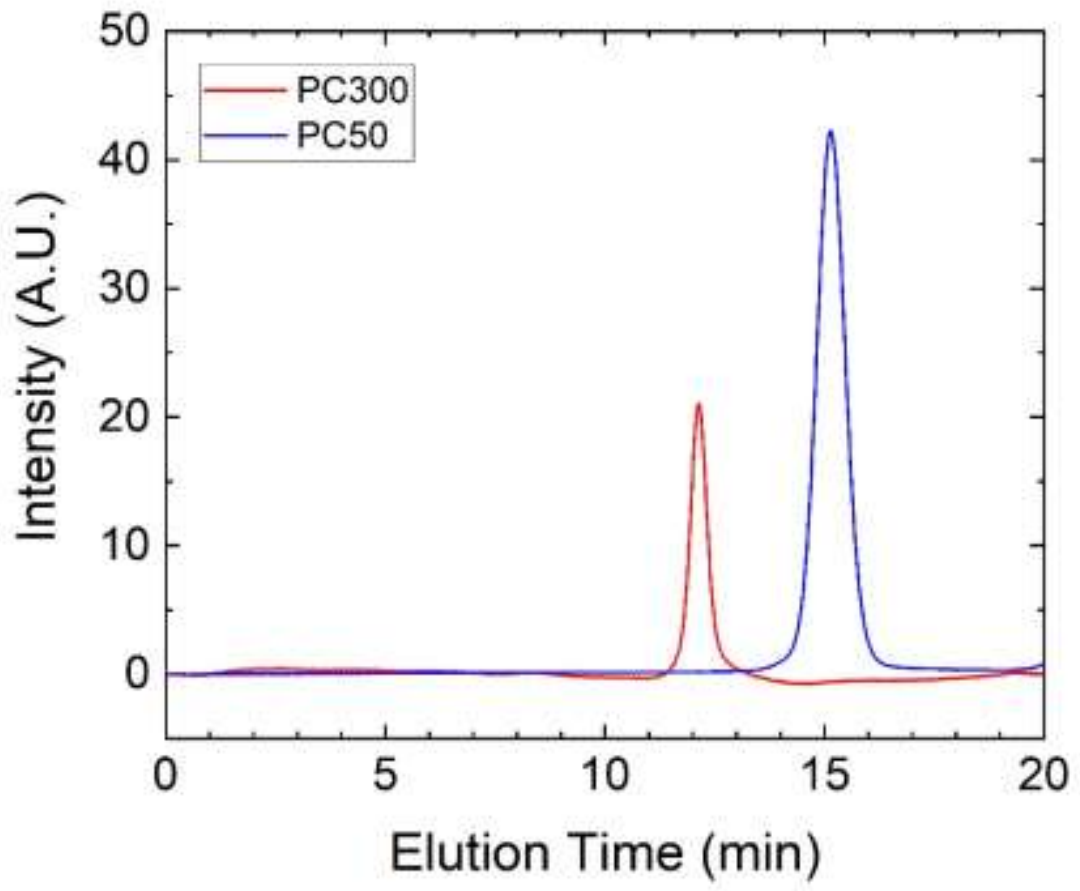

Figure S3. The SEC trace of PC50 and PC 300 measured at $35{ }^{\circ} \mathrm{C}$, where THF was used as eluent. The measured $M_{\mathrm{n}}$ and dispersity of polymer from the PS standard calibration are $13 \mathrm{kDa}$ and 1.03 for PC50, and $76 \mathrm{kDa}$ and 1.05 for PC300, respectively. The results obtained for PC50 are coherent with those from MALDI. 


\section{MALDI-TOF-MS}
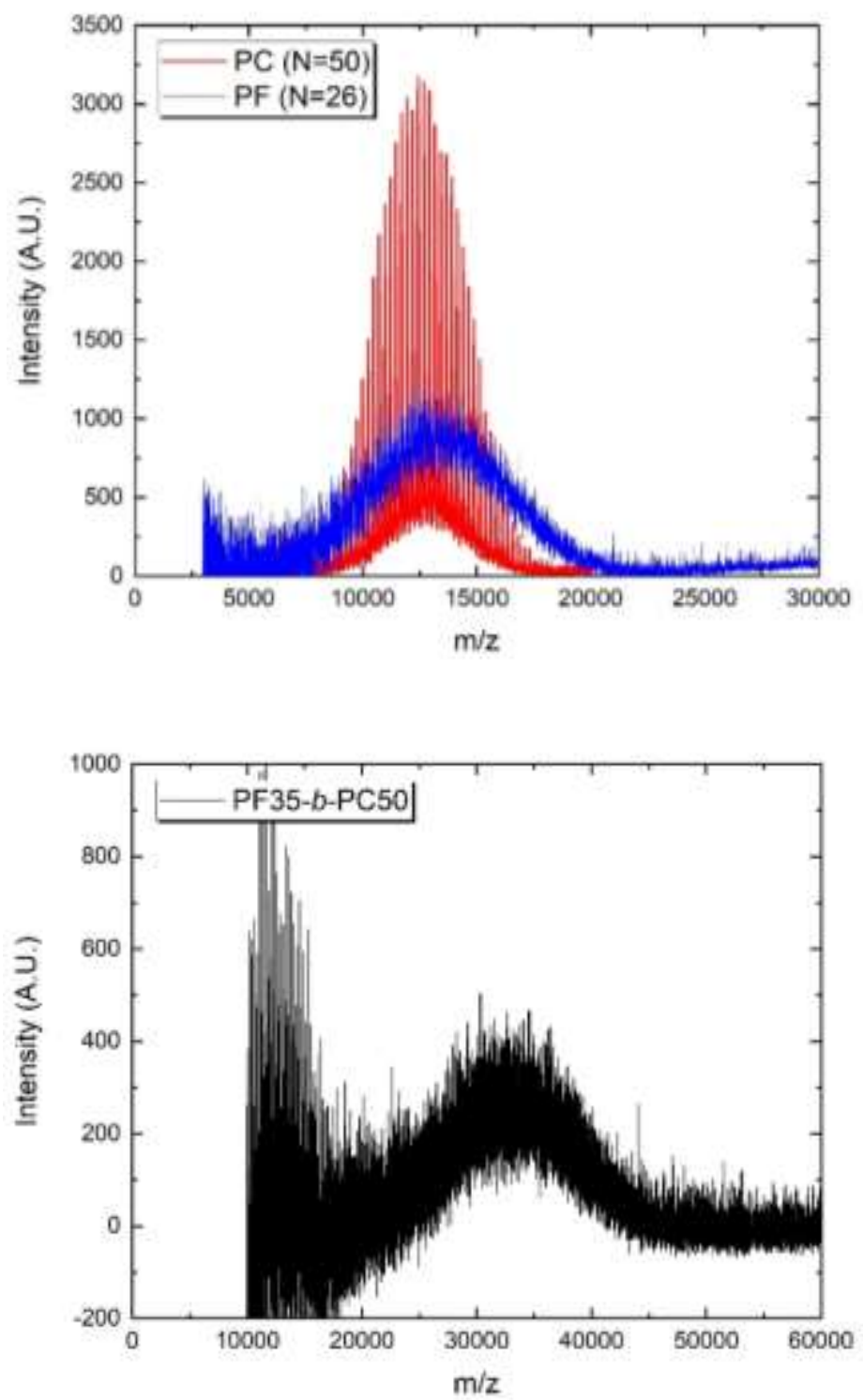

Figure S4. The MALDI traces of PC50, PF26, and PF35-b-PC50. The measured $M_{\mathrm{n}}$ and dispersities are $12 \mathrm{kDa}$ and 1.02 for PC50, and $13 \mathrm{kDa}$ and 1.05 for PF26, and $32 \mathrm{kDa}$ and 1.03 for PF35- $b$-PC50, respectively. 
Table S2. Number-averaged molecular weight $\left(M_{\mathrm{n}}\right)$ and dispersity $(\nexists)$ of homopolymers obtained using NMR ${ }^{\mathrm{a}}$, $\mathrm{SEC}^{\mathrm{b}}$, and $\mathrm{MALDI}{ }^{\mathrm{c}}$. ${ }^{\mathrm{a}} M_{\mathrm{n}}$ was estimated from the monomer conversion.

\begin{tabular}{|cccccc|}
\hline Polymer & $\boldsymbol{M}_{\mathbf{n}}(\mathbf{k D a})^{\mathbf{a}}$ & $\boldsymbol{M}_{\mathbf{n}}(\mathbf{k D a})^{\mathbf{b}}$ & $\boldsymbol{Ð}^{\mathbf{b}}$ & $\boldsymbol{M}_{\mathbf{n}}(\mathbf{k D a})^{\mathbf{c}}$ & $\boldsymbol{\Xi}^{\mathbf{c}}$ \\
\hline PF26 & 12.8 & - & - & 13 & 1.05 \\
PC50 & 12.3 & 13 & 1.03 & 12 & 1.02 \\
PC300 & 73.6 & 76 & 1.05 & - & - \\
\hline
\end{tabular}




\section{Elemental Analysis}

Table S3. Carbon content of polymers obtained using elemental analysis (EA). Each specimen was measured 3 times. ${ }^{\text {a }}$ Degree of polymerization $\left(N_{\mathrm{PF}, \mathrm{n}}\right)$ and $M_{\mathrm{n}}$ of PF block were estimated from the known molecular weight of mother polymer (PC block) and the measured carbon $\mathrm{wt} \%$ of specimen.

\begin{tabular}{|cccc|}
\hline Polymer & $\begin{array}{c}\text { Theoretical } \\
\text { carbon (\% weight) }\end{array}$ & $\begin{array}{c}\text { Experimental } \\
\text { Carbon (\% weight) }\end{array}$ & $\boldsymbol{N}_{\mathbf{P F}, \mathbf{n}}{ }^{\mathbf{a}}$ \\
\hline PF26 & 35.17 & $35.65 \pm 0.09$ & - \\
PC50H & 73.45 & $73.42 \pm 0.06$ & - \\
PC300D & 70.33 & $70.14 \pm 0.09$ & \\
PF35-b-PC50 & - & $50.21 \pm 0.14$ & $34.6 \pm 0.5$ \\
PF56-b-PC50 & - & $46.59 \pm 0.05$ & $56.3 \pm 0.3$ \\
PF79-b-PC50 & - & $44.08 \pm 0.06$ & $78.9 \pm 0.7$ \\
PF90-b-PC300H & - & $59.20 \pm 0.23$ & $85.2 \pm 2.1$ \\
PF90-b-PC300D & - & $57.08 \pm 0.05$ & $90.6 \pm 0.6$ \\
PF120-b-PC300H & - & $56.27 \pm 0.14$ & $117.0 \pm 1.8$ \\
PF120-b-PC300D & - & $54.66 \pm 0.07$ & $120.4 \pm 0.9$ \\
PF180-b-PC300H & - & $51.84 \pm 0.03$ & $186.2 \pm 0.7$ \\
PF180-b-PC300D & - & $51.14 \pm 0.07$ & $180.1 \pm 1.5$ \\
\hline
\end{tabular}




\section{Conformation and Interactions of Core-Block ("PF") Polymer}

\section{A. Surface Energy}

First, the conventional two-liquid analysis (Wu method) was applied for a crude estimation of the contributions from the polar and dispersive surface energies. Table S3 summarizes the results and suggests that the dispersive term of PF polymer dominates the surface characteristics, and as well, the interaction between other substances. The dispersive energy was then characterized using the concept of critical surface energy (Zisman method) which yielded more robust results (Figure S5). Therefore, it is used for further estimation of the solubility parameter and the interaction parameter in relation to THF (Table S4).
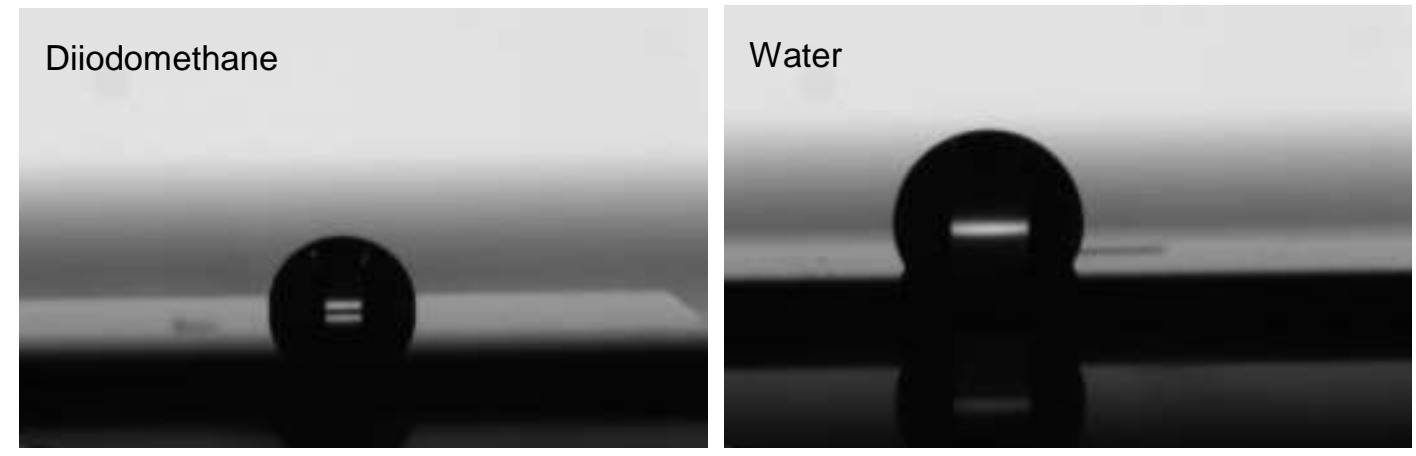

Figure S5. The sessile drop images of test liquids, diiodomethane and water, on PF500-coated Si wafer. 
Table S4. Thermodynamic parameters of PF homopolymer determined from contact angles using the Wu method.

\begin{tabular}{|cc|}
\hline Test Liquid & Contact Angle at $\mathbf{2 5}^{\mathbf{}} \mathbf{C}$ (deg) \\
\hline Water & $119.1 \pm 2.0$ \\
$97.7 \pm 1.0$ \\
\hline Diiodomethane & $14.2 \pm 0.5 \mathrm{mN} / \mathrm{m}$ \\
Total surface energy & $12.9 \pm 1.1 \mathrm{mN} / \mathrm{m}$ \\
$-\quad$ Non-polar surface energy & $1.3 \pm 1.0 \mathrm{mN} / \mathrm{m}$ \\
\hline
\end{tabular}

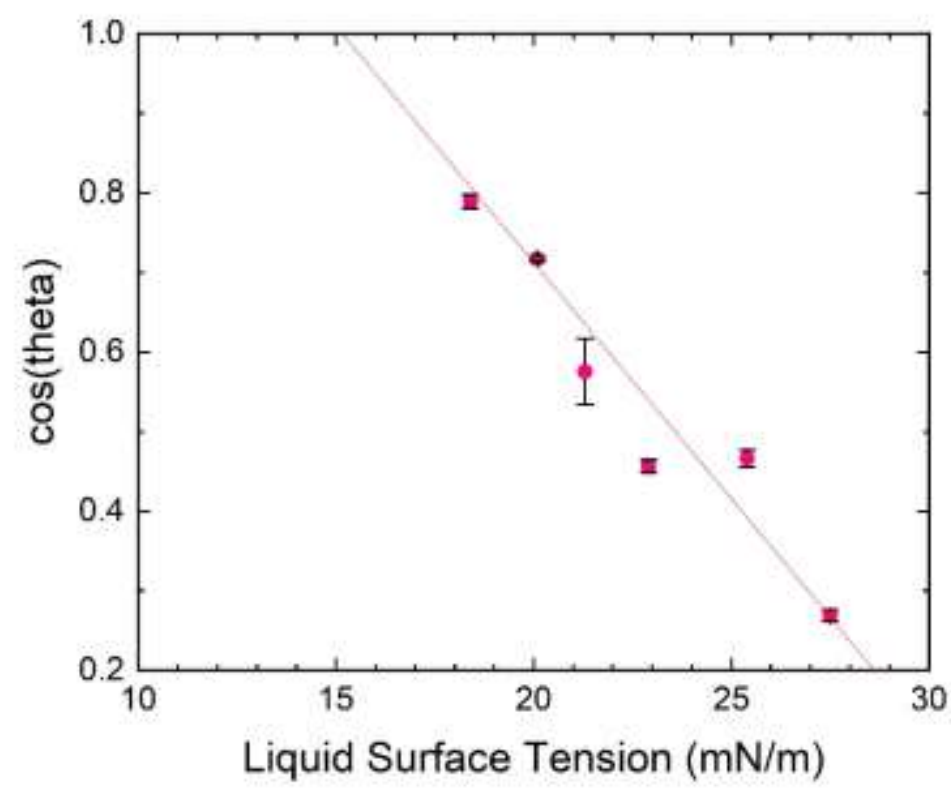

Figure S6. The Zisman plot constructed from contact angles (theta) of different $n$-alkanes (the number of carbon $=6,7,8,9$, and 16) plus cyclohexane. The critical surface energy is estimated as $15.2 \pm 1.1 \mathrm{mN} / \mathrm{m}$ from the linear fit $\left(\mathrm{R}^{2}=0.96\right)$. 
Table S5. Thermodynamic parameters of PF homopolymer determined from the Zisman method.

\begin{tabular}{|ll|}
\hline Critical Surface energy & $15.2 \pm 1.1 \mathrm{mN} / \mathrm{m}$ \\
\hline Solubility Parameter $^{\mathbf{a}}$ & $14.5 \pm 0.4\left(\mathrm{~J} \mathrm{~cm}^{3}\right)^{0.5}$ \\
\hline$\gamma_{\text {PN-THF }^{\mathbf{b}}}$ & $3.6 \pm 0.7 \mathrm{mN} / \mathrm{m}$ \\
\hline$\chi_{\text {PF-THF }^{c}}^{\mathbf{c}}$ & $1.1 \pm 0.1$ \\
\hline
\end{tabular}

${ }^{\mathrm{a}}$ Reference $3 .{ }^{\mathrm{b}}$ Reference $4 .{ }^{\mathrm{c}}$ Calculated from $\gamma_{\mathrm{PN}-\mathrm{THF}}$; see Characterization sub-section in page 3 . 


\section{B. Chain Stiffness}

\section{Estimation of chain stiffness of bottlebrush PF polymers}

The Kuhn segmental length $\left(l_{\mathrm{K}}\right)$ of PF polymers was determined from SAXS conformation study of PF homopolymer $(N=500)$ in nearly theta solvent TFT. Figure S1 shows absolute scaled intensity of PF and the model fits according to the worm-like chain (WLC) model, as well as the Debye model, with known contour length $(N l=31 \mathrm{~nm}) .{ }^{5}$ The fitting was performed using IGOR Pro with NCNR Analysis Macros. ${ }^{6}$ The WLC fit results in $l_{\mathrm{K}}=5.69 \mathrm{~nm}$ and $R_{\mathrm{g}}=17.3 \mathrm{~nm}$ with much better correspondence than that of the Debye function (ideal Gaussian chain), especially at $q<0.02 \AA^{-1}$.

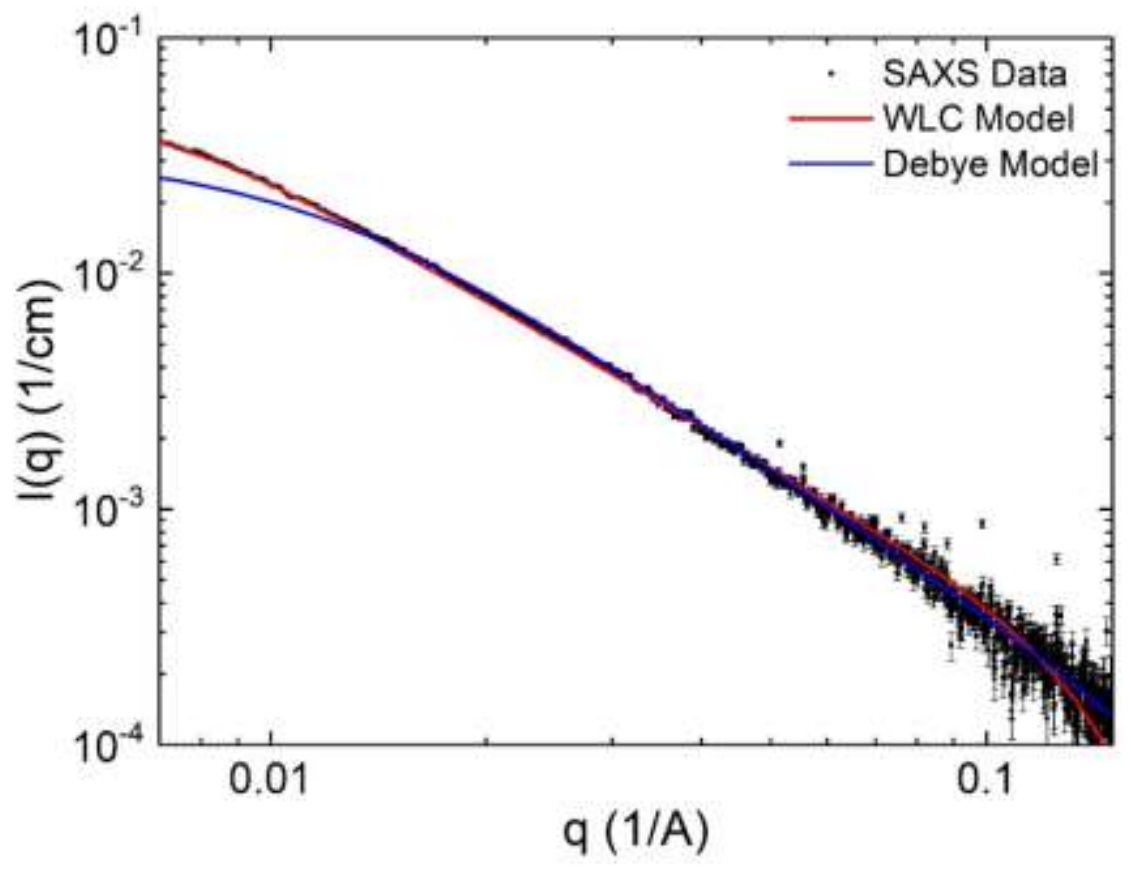

Figure S7. SAXS intensity curve of $2.5 \mathrm{mg} / \mathrm{ml}$ PF homopolymers $(N=500)$ solution with nearly theta solvent $\alpha, \alpha, \alpha$-trifluorotoluene. Data were fitted using either the worm-like chain (WLC) model (red) or the Debye model (blue), respectively. 


\section{Micelle Characterization}

\section{A. TEM}
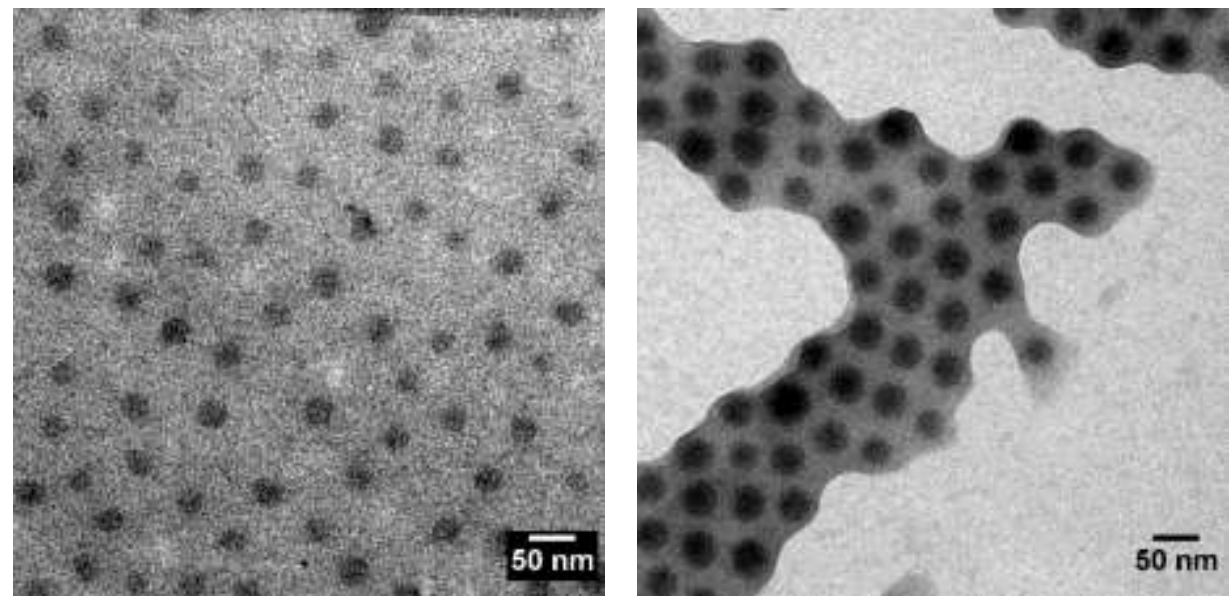

Figure S8. TEM images of dried PF90-PC300H (left) and PF180-PC300H (right) micelles after thermal equilibration. The spherical morphologies with boundary between the core and background are clearly visible. No staining was employed as the high electron density of the fluorine-rich core block provided sufficient density contrast. 


\section{B. DLS}

\section{i. Cumulant Fit Results}

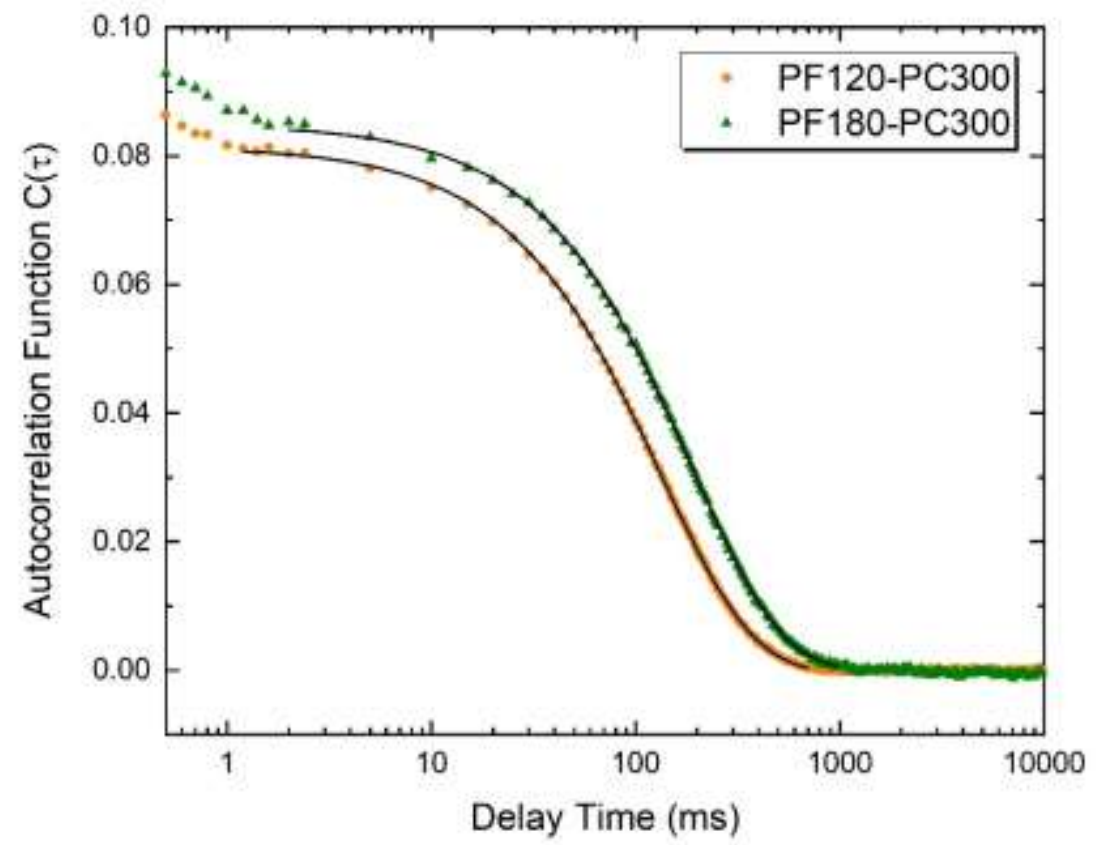

Figure S9. An exemplary display of DLS analysis showing autocorrelation functions of PF120-PC $300 \mathrm{H}$ and PF180-PC300H in micelle form in THF. DLS curves were obtained at the scattering angle of $90^{\circ}$ at $25^{\circ} \mathrm{C}$. The solid lines represent cumulant fit. Highly uniform, structurally isotropic micelles were observed (dispersity $=0.07$ and 0.03 , respectively). 


\section{ii. Scattering Angle Dependence}
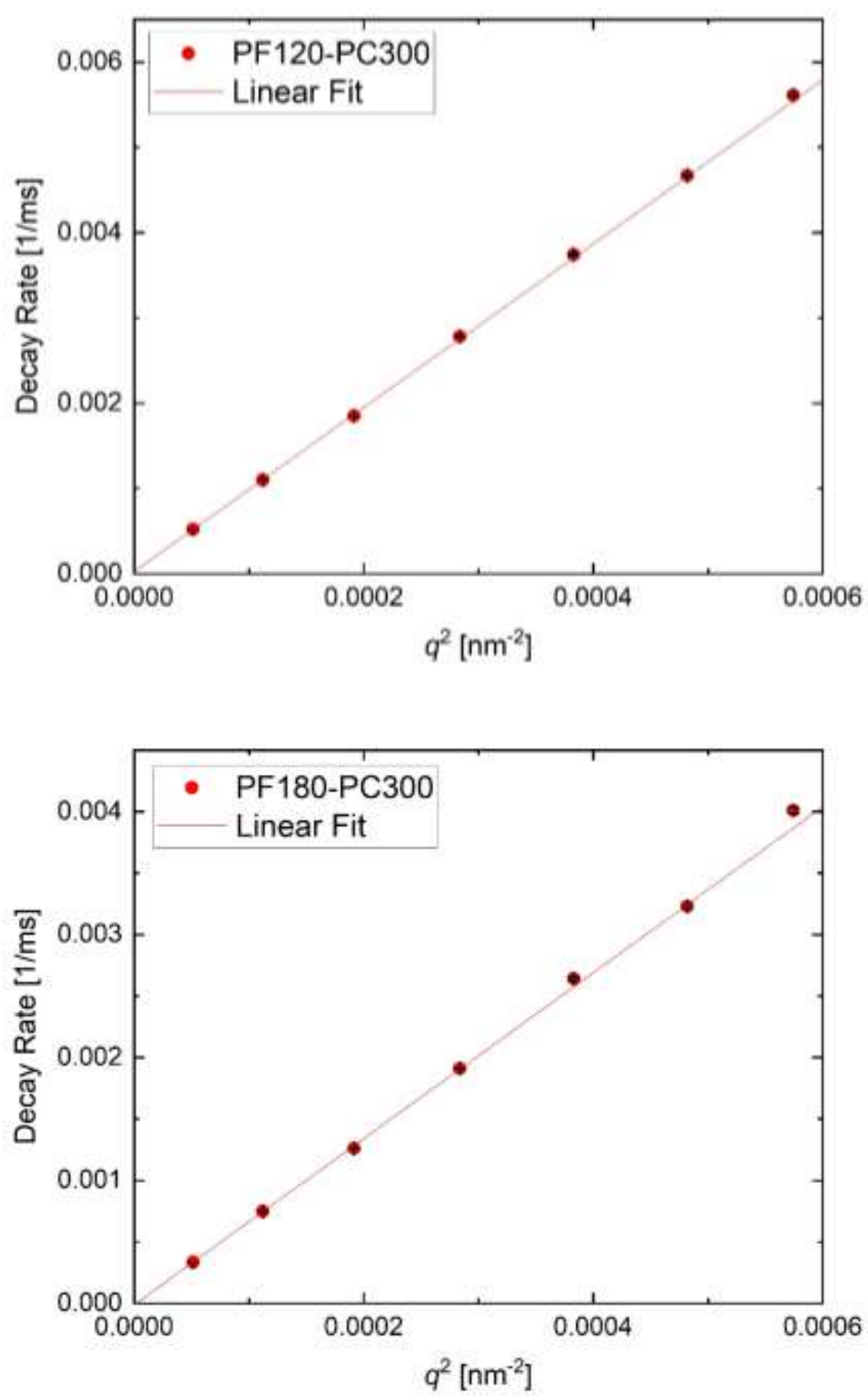

Figure S10. Multi-angle DLS results from exemplary samples of PF120-PC300H and PF180-PC300H micelles. The decay rate $(\Gamma)$ was obtained from the cumulant fitting. Good linearity over whole range of $q^{2}$ indicates the structural isotropy of micelles for both samples, i.e. block copolymers with the longest core blocks used in this study. 


\section{iii. Extrapolation to Infinite Dilution}

Hydrodynamic radius $\left(R_{\mathrm{h}}\right)$ was characterized using dynamic light scattering (DLS) for estimation of the corona thickness of PF- $b$-PC micelles. For each sample, a series of measurements with different concentrations was made to deduce the zero-concentration diffusion coefficient $\left(D_{0}\right)$, which is converted to $R_{\mathrm{h}}$ following the Stokes-Einstein relation, $D_{0}=k_{\mathrm{B}} T / 6 \pi \eta R_{\mathrm{h}}$, where $k_{\mathrm{B}}$ is the Boltzmann constant, $T$ the temperature, and $\eta$ the viscosity of solvent. The resulting hydrodynamic radius and corona thickness for each sample is listed in Table 2 in the manuscript.

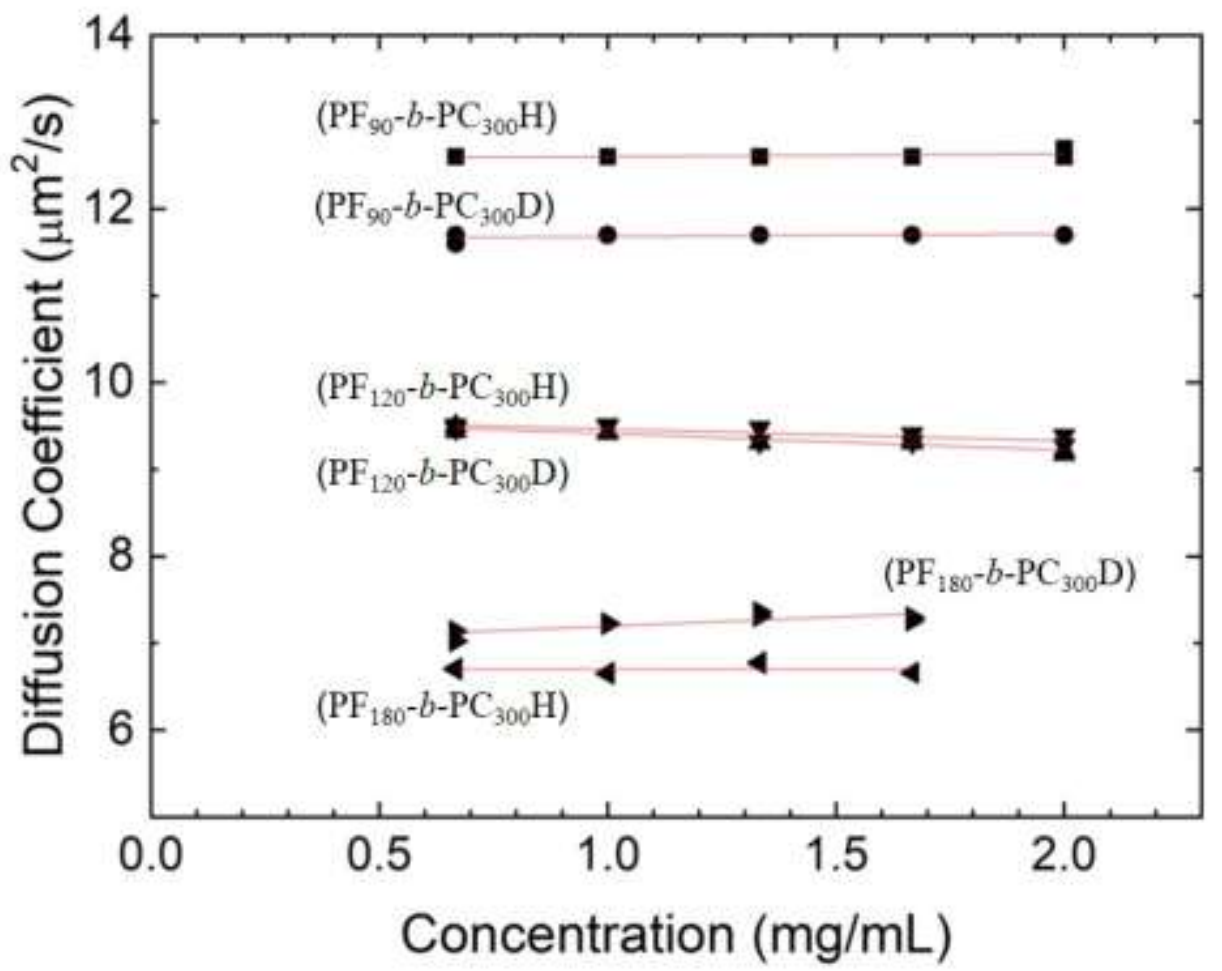

Figure S11. Diffusion coefficients $(D)$ measured from DLS experiments with varying polymer concentrations. Each datapoint was obtained from the cumulant method. The solid lines are linear fits from which the zeroconcentration diffusion coefficient $\left(D_{0}\right)$ was determined and used for the calculation of the hydrodynamic radius $\left(R_{\mathrm{h}}\right)$. 


\section{SLS}

i. Zimm Plots

Table S6. $\mathrm{d} n / \mathrm{d} c$ and SLS results of PF- $b$-PC micelles in THF.

\begin{tabular}{|cccccc|}
\hline Polymer & $\begin{array}{c}\mathbf{d} \boldsymbol{n} / \mathbf{d} \boldsymbol{c} \text { in THF } \\
(\mathbf{m L} / \mathbf{m g})\end{array}$ & $\boldsymbol{M}_{\mathbf{w}, \text { chain }}(\mathbf{k D a})$ & $\boldsymbol{M}_{\mathbf{w}, \mathbf{a g g}}(\mathbf{M D a})$ & $\boldsymbol{N}_{\text {agg }}$ & $\boldsymbol{R}_{\mathbf{g}, \text { mic }}(\mathbf{n m})$ \\
\hline PF90-PC300H & $0.078 \pm 0.005$ & 119 & $10.1 \pm 1.2$ & $85 \pm 10$ & $31 \pm 2$ \\
PF120-PC300H & $0.064 \pm 0.004$ & 132 & $21.9 \pm 2.4$ & $166 \pm 18$ & $42 \pm 2$ \\
PF180-PC300H & $0.047 \pm 0.004$ & 166 & $79.3 \pm 9.2$ & $478 \pm 55$ & $63 \pm 3$ \\
\hline
\end{tabular}

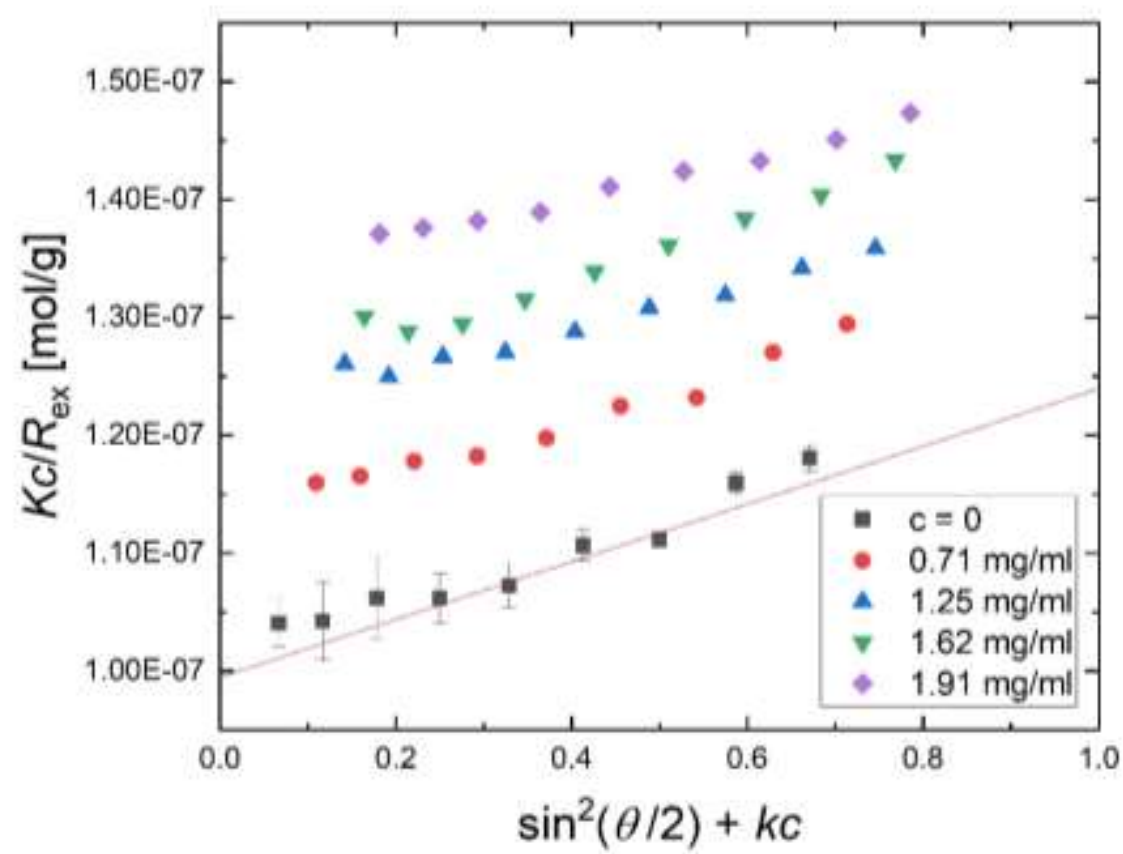

Figure S12. The Zimm plot of PF90-PC300H micelles in THF at $25{ }^{\circ} \mathrm{C}$ with $k=0.06 \mathrm{~mL} / \mathrm{mg}$. The datapoints were first used to extrapolate to $c=0$, and then linear fit (solid line) was applied to yield the $q=0$ extrapolation. 


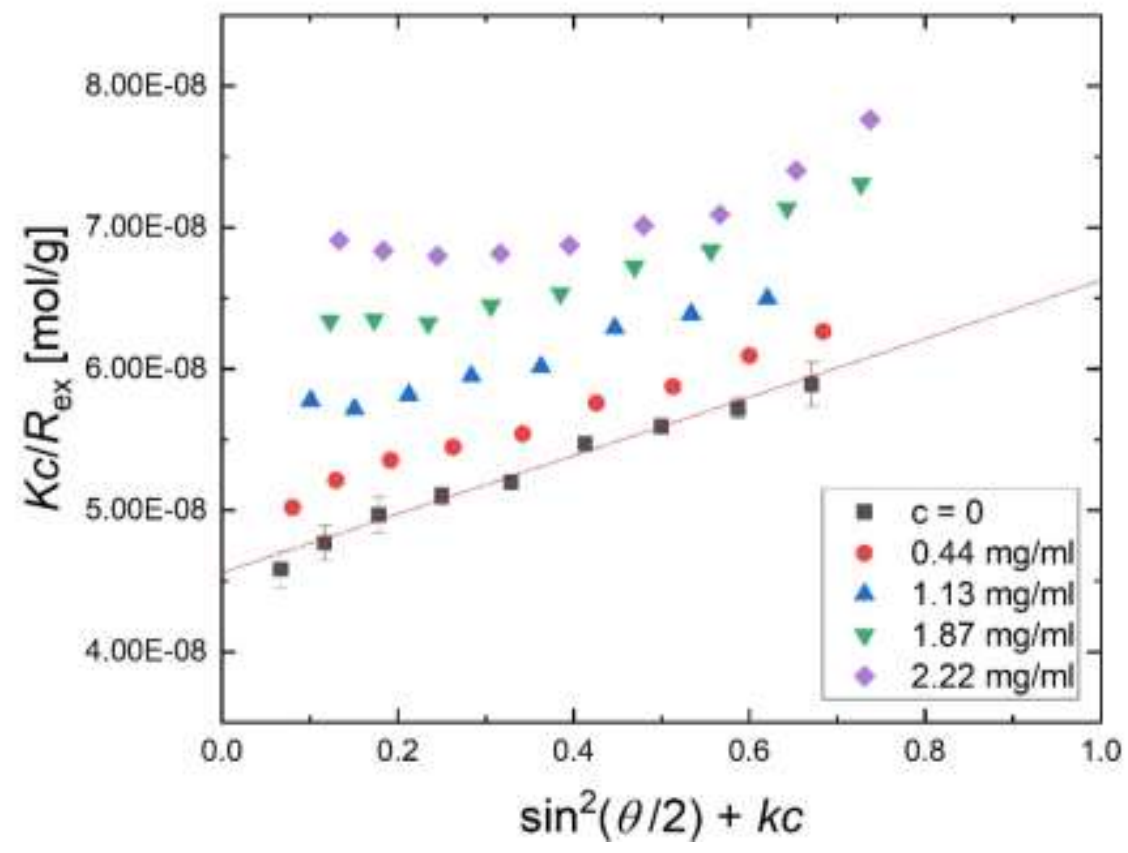

Figure S13. The Zimm plot of PF120-PC300H micelles in THF at $25{ }^{\circ} \mathrm{C}$ with $k=0.03 \mathrm{~mL} / \mathrm{mg}$. The datapoints were first used to extrapolate to $c=0$, and then linear fit (solid line) was applied to yield the $q=0$ extrapolation.

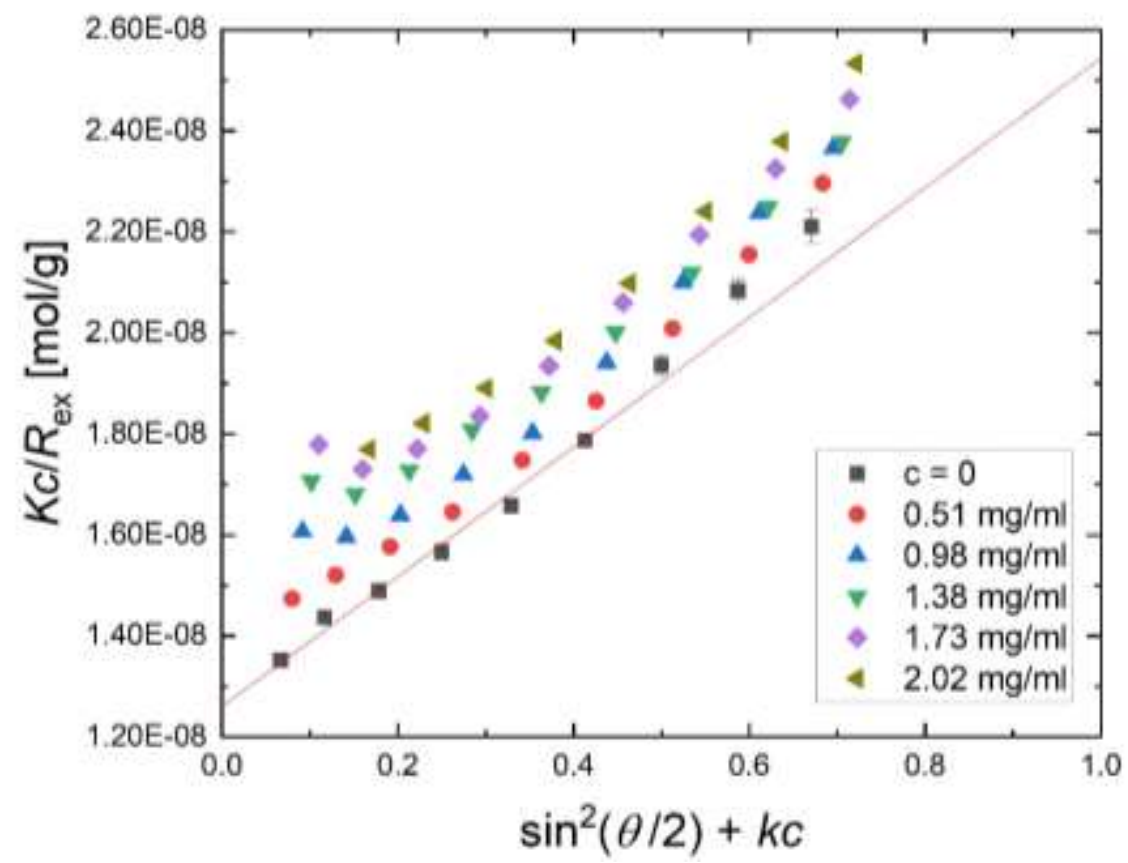

Figure S14. The Zimm plot of PF180-PC300H micelles in THF at $25{ }^{\circ} \mathrm{C}$ with $k=0.025 \mathrm{~mL} / \mathrm{mg}$. The datapoints were first used to extrapolate to $c=0$, and then linear fit (solid line) was applied to yield the $q=0$ extrapolation. 


\section{ii. Aggregation Numbers $\left(N_{\text {agg }}\right)$}

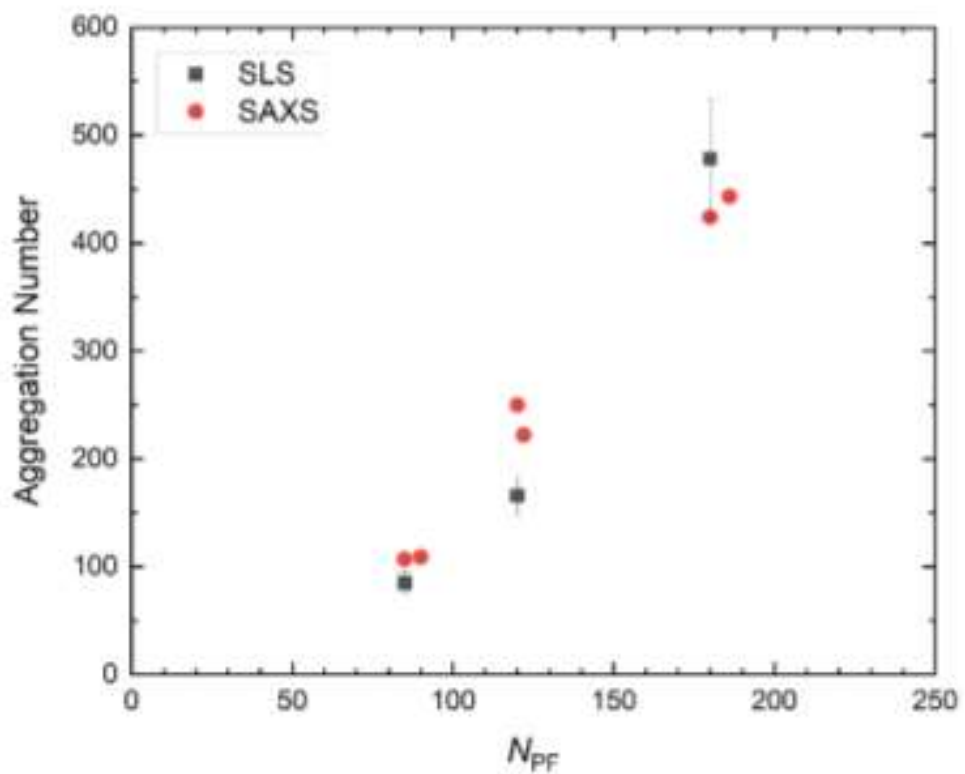

Figure S15. The aggregation numbers of PC300 series (i.e., $N_{\mathrm{PC}}=300$ ) as a function of the number of repeat units in the core block $\left(N_{\mathrm{PF}}\right)$. Values were determined from SLS and SAXS. 


\section{References}

1. Cho, Y.; Kim, Y.; Choi, T.-L.; Lim, J.; Char, K. Swelling-induced pore generation in fluorinated polynorbornene block copolymer films. Polymer Chemistry 2018, 9, 3536-3542.

2. Pedersen, J. S.; Svaneborg, C.; Almdal, K.; Hamley, I. W.; Young, R. N. A small-angle neutron and X-ray contrast variation scattering study of the structure of block copolymer micelles: Corona shape and excluded volume interactions. Macromolecules 2003, 36, 416-433.

3. Jia, L.; Shi, B. A New Equation between Surface Tensions and Solubility Parameters without Molar Volume Parameters Simultaneously Fitting Polymers and Solvents. Journal of Macromolecular Science, Part B 2011, 50, $1042-1046$

4. Wu, S. Calculation of interfacial tension in polymer systems. J. Polymer Sci.: Part C 1971, 34, 19-30.

5. Chen, W. R.; Butler, P. D.; Magid, L. J. Incorporating intermicellar interactions in the fitting of SANS data from cationic wormlike micelles. Langmuir 2006, 22, 6539-6548.

6. Kline, S. R. Reduction and Analysis of SANS and USANS Data using Igor Pro. J Appl. Cryst. 2006, $39,895$.

7. Sprouse, D.; Jiang, Y.; Laaser, J. E.; Lodge, T. P.; Reineke, T. M. Tuning Cationic Block Copolymer Micelle size by $\mathrm{pH}$ and Ionic Strength. Biomacromolecules 2016, 17, 2849-2859. 